\title{
Odd Operators and Spinor Algebras in Lattice Statistics: n-Point Functions for the Rectangular Ising Model *
}

\author{
D. B. Abraham
}

Department of Theoretical Chemistry, Oxford University, Oxford OX1 3TG, England

\begin{abstract}
An extension is given of the development by Schultz et al. [3] of the pioneering work of Kaufman and Onsager [2] on the planar Ising ferromagnet. This involves a novel form of Wick's theorem [19] for fermions. In subsequent papers the method will be applied to determine the $n$-point functions and to evaluate rigorously critical indices.
\end{abstract}

\section{Introduction}

The algebraic method of computing quantities in the Ising model requires the spectral decomposition of the transfer matrix and the evaluation, in the basis generated thereby, of matrix elements of appropriate operators. The purpose of this paper and the following one is to give a complete determination of all such matrix elements for the planar ferromagnet with nearest neighbour interactions and subject to cyclic boundary conditions. The techniques pioneered by Onsager [1] and Kaufman [2], together with their subsequent development by Schultz et al. [3], will be extended. Thus the present work is quite unlike that of $\mathrm{Wu}$ and coworkers [4] in which combinatorial concepts and Pfaffians are introduced at the outset. Further, the results given here are rigorous. The principal application reported here is the determination of $n$-point functions, [5], also obtained by McCoy et al. [6]. The 4-point function has been investigated in some detail by $\mathrm{Au}$ Yang [7]. Previous applications of these results are the proof that the susceptibility divergence exponents are $\gamma=\gamma^{\prime}=7 / 4[8]$ and the investigation of the interface between coexisting pure phases [9]. To put the results in context, the Ising model will now be defined: let $\mathbb{Z}^{d}$ generate the $d$-dimensional hypercubical crystal lattice with unit edge, the Cartesian coordinates of each lattice site, called a vertex, being $\boldsymbol{r}=\left(r_{1} \ldots r_{d}\right), r_{i} \in \mathbb{Z}$. At each vertex $\boldsymbol{r}$ there is a $\operatorname{spin} \sigma(\boldsymbol{r})= \pm 1$. A spin configuration $\{\sigma\}$ on a sub-box $\Lambda$ is defined by specifying $\sigma(\boldsymbol{r})$ for each $\boldsymbol{r} \in \Lambda$, and

* Supported by the Fonds National Suisse de la Recherche Scientifique, by the National Science Foundation Grant No. PHY76-17191, and by the National Research Council of Canada Grant No. NRC A9344 
the collection of all such configurations is the phase space $\mathbb{P}(\Lambda)$. The configuration energy $E_{\Lambda}(\{\sigma\})$ is a function from $\mathbb{P}(\Lambda)$ to $\mathbb{R}$ given by

$$
E_{\Lambda}(\{\sigma\})=-\sum J\left(\boldsymbol{r}_{1}-\boldsymbol{r}_{2}\right) \sigma\left(\boldsymbol{r}_{1}\right) \sigma\left(\boldsymbol{r}_{2}\right)-H \sum \sigma(\boldsymbol{r})-\mathscr{B}_{\Lambda} .
$$

In the above $J\left(\boldsymbol{r}_{1}-\boldsymbol{r}_{2}\right)$ is a non-negative translationally invariant coupling and $J(\boldsymbol{r})=0$ unless $\boldsymbol{r}= \pm e_{i}$ for some $i$ where $\left(e_{i}\right)_{j}=\delta_{i j}$, the Kronecker delta. The latter requirement stipulates that the interactions are of nearest-neighbour type. If $J(\boldsymbol{r}) \geqq 0$ then the system is termed ferromagnetic. The second terms is a polarisation due to a magnetic field $H$. The summations are over all $\boldsymbol{r}_{i} \in \Lambda$. Finally $\mathscr{B}_{A}$ is a boundary term; it might specify, for instance, that all spins on the surface $\partial \Lambda$ of $\Lambda$ are +1 , or that there are toroidal boundary conditions so that spins of opposite sides of $\Lambda$ are coupled by a $J$. The probability of a configuration on $\Lambda$ is given by:

$$
p_{\Lambda}(\{\sigma\})=Z_{\Lambda}^{-1} \exp -\beta E_{\Lambda}(\{\sigma\}),
$$

where $\beta=1 / k_{B} T, T$, and $k_{B}$ being the absolute temperature and Boltzmann's constant respectively; $Z_{A}$ is the canonical partition function which normalizes (1.2). The dependence of $p_{\Lambda}$ on the boundary conditions $\mathscr{B}_{\Lambda}$ is crucial for an understanding of phase transitions in the following sense: let $A \subset \mathbb{Z}^{d}$ be a subset with diameter $d(A)<\infty$, and let $O_{A}: \mathbb{P}\left(\mathbb{Z}^{d}\right) \rightarrow \mathbb{R}$ be a function of support $A$, this is, let it be independent of $\sigma_{i}$ if $i \in \mathbb{Z}^{d} \backslash A$. Consider the expectation values with respect to (1.2) denoted $\left\langle O_{A}\right\rangle_{A, \mathscr{B}_{A}}$ and, finally their limiting behaviour

$$
\left\langle O_{A}\right\rangle_{\mathscr{B}}=\lim _{\Lambda \rightarrow \infty}\left\langle O_{A}\right\rangle_{A, \mathscr{B}_{A}},
$$

where the Euclidean distance $d(\Lambda, \partial \Lambda) \rightarrow \infty$ as $\Lambda \rightarrow \infty$ in the sense of van Hove. We shall be particularly interested in the dependence of $\left\langle O_{A}\right\rangle_{\mathscr{B}}$ on the sequence of boundary conditions. If there is dependence for some $\left\{\mathscr{B}_{A}\right\}$, then we have a phase transition, by definition [10]. For instance, it has been shown that for the planar ferromagnet with $A=(0,0)$ and $\mathscr{B}_{+(\text {resp-) }}=\left\{\sigma_{i}=+1\right.$ (resp -1) $\left.\forall i \in \partial \Lambda\right\}$ that $\left.-\left\langle\sigma_{0}\right\rangle_{-}=\left\langle\sigma_{0}\right\rangle_{+}\right\rangle 0$ for all $\beta>\beta_{c}$ where $\beta_{c}^{-1}$ is Onsager's critical temperature. By placing + spins round the upper half and - spins round the lower half, phase separation can be studied. The relevance of matrix element computations to this is discussed in [9]. The first problem is, then, to write down quantities in terms of transfer matrices [11]. This is done for two simple examples for dimension $d=2$ to fix notation.

Let $\Lambda$ be a ractangle with $N$ rows and $M$ columns which is wrapped on a torus by specifying a suitable $\mathscr{B}_{\Lambda}$ in (1.1). Let $x_{n}$ denote a configuration of the $M$ spins on row $n$, written in a binary form: $\left(x_{n}\right)_{i}= \pm 1, i=1, \ldots, M$; there are $2^{M}$ such configurations. Define the matrices $T_{1}$ and $T_{2}$ by:

$$
\begin{aligned}
& T_{1}\left(x_{n}, x_{n+1}\right)=\exp K_{1} \sum_{i=1}^{M}\left(x_{n}\right)_{i}\left(x_{n+1}\right)_{i} \\
& T_{2}\left(x_{n}, x_{n+1}\right)=1\left(x_{n}, x_{n+1}\right) \exp \sum_{1}^{M}\left(K_{2}\left(x_{n}\right)_{i}\left(x_{n}\right)_{i+1}+h\left(x_{n}\right)_{i}\right),
\end{aligned}
$$

where $K_{j}=\beta J_{j}, h=\beta H$. 
Let the state of the top and bottom rows be described by the vectors $p_{1}$ and $p_{N}$. Then the partition function for such a situation is given by

$$
\begin{aligned}
Z= & \sum_{x_{1}, x_{1}^{\prime} ; \ldots ; x_{N} x_{N^{\prime}}} p\left(x_{1}^{\prime}\right) T_{2}\left(x_{1}^{\prime}, x_{1}\right) \\
& \cdot \prod_{1}^{N-1}\left(T_{1}\left(x_{j}, x_{j}^{\prime}\right) T_{2}\left(x_{j}^{\prime}, x_{j+1}\right)\right) p_{N}\left(x_{N}\right)
\end{aligned}
$$

which is in the form of a matrix product:

$$
Z=p_{1}^{T} T_{2}\left(T_{1} T_{2}\right)^{N-1} p_{N}
$$

For a special choice of $O_{A}$ and $\mathscr{B}$ in (1.3) consider the $n$-point function for toroidal boundary conditions on the lattice $\Lambda$, denoted $\left\langle\sigma\left((\boldsymbol{r})_{n}\right)\right\rangle_{t, N M}$. Here $\left(\boldsymbol{r}_{n}\right)=\boldsymbol{r}_{1}, \ldots, \boldsymbol{r}_{n}$ where $r_{i} \in \mathbb{Z}^{2}$ gives the position of the $i^{\text {th }}$ point. The coordinates $x_{i}$ in the direction of transfer can always be ordered so that $x_{i} \leqq x_{i+1}$ for $i=1, \ldots, n-1$. Then

$$
\left\langle\sigma\left((\boldsymbol{r})_{n}\right)\right\rangle_{t, N M}=Z_{t}^{-1} \operatorname{Tr}\left\{T_{2}\left(T_{1} T_{2}\right)^{x_{1}} \sigma\left(y_{1}\right)\left(T_{1} T_{2}\right)^{x_{2}-x_{1}} \sigma\left(y_{n}\right)\left(T_{1} T_{2}\right)^{N-x_{n}}\right\}
$$

with

$$
Z_{t}=\operatorname{Tr}\left(T_{1} T_{2}\right)^{N}
$$

The layout of this paper is as follows : in Section II notation will be established and the standard results for the spectrum of the symmetrised transfer matrix with zero field will be given. In the following sections the theory of the matrix elements will be presented. There are appendices which give some relevant results from the theory of semi-infinite Töplitz forms and of Wiener-Hopf factorisation.

\section{Notation}

Consider the Hilbert space $\mathscr{H}_{M}$ which is a tensor product of $M$ two-dimensional Hilbert spaces $\mathscr{H}$; this is denoted by $\mathscr{H}_{M}=\bigotimes_{1}^{M} \mathscr{H}$. Any linear operator on this space can be expressed in terms of the Pauli spin operators

$$
\sigma_{j}^{\alpha}=\bigotimes_{1}^{j-1} 1 \otimes \sigma^{2} \bigotimes_{j+1}^{M} 1
$$

where $\sigma^{\alpha}(\alpha=x, y, z)$ in a particular basis for $\mathscr{H}$ have the matrix representation

$$
\sigma^{x}=\left(\begin{array}{ll}
0 & 1 \\
1 & 0
\end{array}\right), \quad \sigma^{y}=\left(\begin{array}{cc}
0 & -i \\
i & 0
\end{array}\right), \quad \sigma^{z}=\left(\begin{array}{rr}
1 & 0 \\
0 & -1
\end{array}\right) .
$$

The $\sigma_{j}^{\alpha}$ have the usual commutation relations.

Returning to (1.4) at seq., let us regard $T_{j}, j=1,2$ as matrix representatives of mapping of $\mathscr{H}_{M}$ onto itself; further, let the configuration of each row be an eigenvector of the $\sigma_{j}^{x}, j=1, \ldots, M$. Then we have

$$
T_{1} \rightarrow V_{1}=\left(2 \sinh 2 K_{1}\right)^{M / 2} \exp -K_{1}^{*} \sum_{1}^{M} \sigma_{j}^{z} .
$$


The symbol $T \rightarrow V$ means that $T$ is a matrix representative of the operator $V$. The function $K_{1}^{*}$ is defined as an involution on $K_{1}$ by

$$
\exp -2 K_{1}^{*}=\tanh K_{1} \text {. }
$$

Finally

$$
T_{2} \rightarrow V_{2} V_{3}
$$

with

$$
\begin{aligned}
& V_{2}=\exp K_{2} \sum_{1}^{M} \sigma_{j}^{x} \sigma_{j+1}^{x} \\
& V_{3}=\operatorname{exph} \sum_{1}^{M} \sigma_{j}^{x} .
\end{aligned}
$$

In the following we shall consider the symmetrised forms

$$
V=V_{3}^{1 / 2} V_{2}^{1 / 2} V_{1} V_{2}^{1 / 2} V_{3}^{1 / 2}
$$

and

$$
V^{\prime}=V_{1}^{1 / 2} V_{3}^{1 / 2} V_{2} V_{3}^{1 / 2} V_{1}^{1 / 2}
$$

which are self-adjoint and therefore have a spectral decomposition.

The symmetry group of $V$ and $V^{\prime}$ contains as a subgroup the translational group $\mathscr{T}_{M}$ of order $M$ generated by the translation $T$ which is defined as follows:

$$
\begin{aligned}
& T \sigma_{j}^{\alpha} T^{-1}=\sigma_{j-1}^{\alpha}, \quad 2 \leqq j \leqq M \\
& T \sigma_{1}^{\alpha} T^{-1}=\sigma_{M}^{\alpha}
\end{aligned}
$$

for $\alpha=x, y, z$. In order to exploit this symmetry, and at the same time retain tractable commutation relations, it is convenient to apply the Jordan-Wigner transformation [4] to (2.1): define

$$
f_{n}^{+}=\prod_{1}^{n-1}\left(-\sigma_{j}^{z}\right)\left(\sigma_{n}^{x}+i \sigma_{n}^{y}\right) \text {. }
$$

Then the $f_{n}$ have Fermi anti-commutation relations

$$
\left[f_{n}, f_{m}\right]_{+}=0, \quad\left[f_{n}, f_{m}^{+}\right]_{+}=\delta_{n m} .
$$

Spinors $\Gamma_{j}$ may be associated through the relations

$$
\Gamma_{2 j-1}=f_{j}^{+}+f_{j}, \quad \Gamma_{2 j}=-i\left(f_{j}^{+}-f_{j}\right) .
$$

Evidently $\left[\Gamma_{j}, \Gamma_{k}\right]_{+}=2 \delta_{j k}$; the $\Gamma_{j}$ generate a Clifford algebra [12] on $\mathscr{H}_{M}$.

The vacuum state $|0\rangle$ in $\mathscr{H}_{M}$ is defined by

$$
f_{n}|0\rangle=0 \forall n=1, \ldots, M
$$

and a basis is given by the vectors

$$
|0\rangle, \quad f_{n}^{+}|0\rangle, \quad f_{n}^{+} f_{m}^{+}|0\rangle, \ldots .
$$


This can be reduced according to the group $\mathscr{T}_{M}$ as follows:

Theorem 1. Let $F_{k}^{+}$be defined by

$$
F_{k}^{+}=M^{-1 / 2} \sum_{1}^{M} e^{i k m} f_{m}^{+} .
$$

Then a sufficient condition that

$$
T F_{k_{1}}^{+} \ldots F_{k_{r}}^{+}|0\rangle=e^{i k} F_{k_{1}}^{+} \ldots F_{k_{r}}^{+}|0\rangle
$$

is that $k=\sum_{1}^{r} k_{j}$, where

$$
\operatorname{expiMk_{j}}=(-1)^{r-1}, \quad j=1, \ldots, r .
$$

Proof. See [13]. Note that wave numbers are required which actually give characters of the cyclic double group.

For convenience, we shall adopt the notations $\alpha$ and $\beta$ for wavenumbers; where $1=\exp i M \alpha=-\exp i M \beta$. We shall also define the sets $S_{M}^{ \pm}$by

$$
S_{M}^{ \pm}=\left\{z: z^{M}=\mp 1\right\}
$$

Theorem 1 motivates the decomposition of $\mathscr{H}_{M}$ into orthogonal subspaces $\mathscr{H}_{M}^{ \pm}$ by the projectors

$$
Q_{ \pm}=(1 \pm P) / 2
$$

where

$$
P=\prod_{1}^{M}\left(-\sigma_{j}^{z}\right)
$$

detects the parity of $r$ for any state $F_{k_{1}}^{+} \ldots F_{k_{r}}^{+}|0\rangle$. Since we have

$$
F_{\beta}^{+}=\sum_{\alpha} F_{\alpha}^{+}\langle\alpha \mid \beta\rangle,
$$

where the transformation function is given by

$$
\langle\alpha \mid \beta\rangle=2 / M(\exp i(\alpha-\beta)-1)
$$

the bases generated by either the $F_{\alpha}^{+}$or the $F_{\beta}^{+}$are equally valid in principle; but in $\mathscr{H}_{M}^{+}$(resp. $\mathscr{H}_{M}^{-}$) we should use $F_{\beta}^{+}$(resp. $F_{\alpha}^{+}$). It is vital to note that

$$
\left[F_{k_{1}}, F_{k_{2}}\right]_{+}=0, \quad\left[F_{k_{1}}, F_{k_{2}}^{+}\right]_{+}=\delta_{k_{1} k_{2}}
$$

provided exp $i k_{j} \in S_{M}^{+}$for both $j=1,2$; or exp $i k_{j} \in S_{M}^{-}$. But from (2.20), (2.21) we have

$$
\left[F_{\beta}^{+}, F_{\alpha}\right]_{+}=\langle\alpha \mid \beta\rangle .
$$

Thus canonicality is only preserved under the transformation (2.14) if both momenta have the same type. 
Any operator $A$ on $\mathscr{H}_{M}$ can be written as

$$
A=A_{o}+A_{e},
$$

where

$$
\left[A_{e}, P\right]_{-}=\left[A_{0}, P\right]_{+}=0 .
$$

Odd operators $A_{o}$ only have non-zero matrix elements between $\mathscr{H}_{M}^{+}$and $\mathscr{H}_{M}^{-}$. A typical example encountered in (2.38) is $\sigma_{n}^{x}$. On the other hand, operators which belong to the even subalgebra $\mathfrak{U}_{e}$ of $\mathfrak{U}$ can be decomposed by $P$ into parts which operate in $\mathscr{H}_{M}^{+}$and in $\mathscr{H}_{M}^{-}$. A typical element of $\mathfrak{U}_{e}$ is $V$, for which

$$
V=V_{+} Q_{+}+V_{-} Q_{-} .
$$

The vital point about $V_{ \pm}$is that, unlike $V$, they are quadratic in the Fermi operators and may thus be brought to diagonal form by canonical transformations. The associated diagonal form are

$$
V_{ \pm}=\left(2 \sinh 2 K_{1}\right)^{M / 2} \exp -\frac{1}{2} \sum \gamma(k)\left(2 G_{k}^{+} G_{k}-1\right),
$$

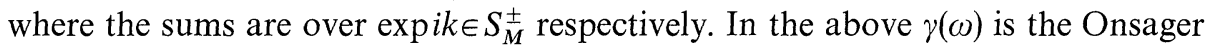
function [7]; it is the root of the equation

$$
\cosh \gamma(\omega)=\cosh 2 K_{1}^{*} \cosh 2 K_{2}-\sinh 2 K_{1}^{*} \sinh 2 K_{2} \cos \omega
$$

which is positive for the real $\omega$. The $G_{k}^{+}$are given by a Bogoliubov-Valatin transformation [14]:

$$
G_{k}^{+}=\cos \theta(k) F_{k}^{+}-i \sin \theta(k) F_{-k} .
$$

Provided that $\theta(k)$ satisfies the relationship $\theta(k)=-\theta(-k) \bmod \pi$, this is canonical under the same restrictions as in (2.22).

Otherwise there is a linear dependence relation from (2.20) and (2.21)

$$
G_{\beta}=\sum_{\alpha}\langle\beta \mid \alpha\rangle\left(\cos \left(\theta_{\beta}-\theta_{\alpha}\right) G_{\alpha}+i \sin \left(\theta_{\beta}-\theta_{\alpha}\right) G_{-\alpha}^{+}\right) .
$$

In the rest of this paper the following notation will be used:

$$
\Theta\left(e^{i \omega}\right)=\exp -2 i \theta(\omega) \text {. }
$$

For $V^{\prime}$, one has the same dispersion function $\gamma(\omega)$ as for $V$ but

$$
\Theta(z)=\frac{1}{(A B)^{1 / 2}}\left[\frac{(z-A)(z-B)}{\left(z-A^{-1}\right)\left(z-B^{-1}\right)}\right]^{1 / 2}
$$

whereas for $V$ one has

$$
\Theta(z)=-\left(\frac{A}{B}\right)^{1 / 2} \frac{1}{z}\left[\frac{(z-B)\left(z-A^{-1}\right)}{\left(z-B^{-1}\right)(z-A)}\right]^{1 / 2},
$$

where

$$
A=\operatorname{coth} K_{1}^{*} \operatorname{coth} K_{2}, \quad B=\tanh K_{1}^{*} \operatorname{coth} K_{2} .
$$


Provided $K_{1}^{*}=K_{2}$ (the Onsager critical temperature) $\Theta(z)$ has a pair of branch points inside the unit circle and a pair outside. That sheet of the Riemann surface is selected for which $\Theta(1)=-1$ if $B>1$, but for which $\Theta(1)=+1$ if $B<1$. Let the vectors $\left|\hat{\Phi}_{ \pm}\right\rangle$be defined by

$$
\left|\hat{\Phi}_{ \pm}\right\rangle=\prod\left(\cos \theta(k)+i \sin \theta(k) F_{-k}^{+} F_{k}^{+}\right)|0\rangle,
$$

where exp $i k \in S_{M}^{ \pm}$respectively and $0<k<\pi, M$ even. The vacua for (2.28), $\left|\Phi_{ \pm}\right\rangle$, are given by the equations

$$
G_{\alpha}\left|\Phi_{-}\right\rangle=G_{\beta}\left|\Phi_{+}\right\rangle=0
$$

Then clearly $\left|\Phi_{+}\right\rangle=\left|\hat{\Phi}_{+}\right\rangle$for all $B \neq 1$

but

$$
\left|\Phi_{-}\right\rangle=\left|\hat{\Phi}_{-}\right\rangle\left(\operatorname{resp} . F_{0}^{+}\left|\hat{\Phi}_{-}\right\rangle\right)
$$

if $B<1$ (resp. $B>1$ ). We assume $M$ even; thus $-1 \in S_{M}^{-}$.

The spectra of (2.2) are:

$$
\begin{aligned}
& B>1: \\
& \mathscr{H}_{M}^{+}:\left|\Phi_{+}\right\rangle G_{\beta_{1}}^{+} G_{\beta_{2}}^{+}\left|\Phi_{+}\right\rangle \\
& \mathscr{H}_{M}^{-}:\left|\Phi_{-}\right\rangle G_{\alpha_{1}}^{+} G_{\alpha_{2}}^{+}\left|\Phi_{-}\right\rangle .
\end{aligned}
$$

There will be an asymptotic double degeneracy corresponding to coexistence of 2 pure phases [15].

$$
\begin{aligned}
& B<1: \\
& \mathscr{H}_{M}^{+}:\left|\Phi_{+}\right\rangle G_{\beta_{1}}^{+} G_{\beta_{2}}^{+}\left|\Phi_{+}\right\rangle \\
& \mathscr{H}_{M}^{-}: G_{\alpha}^{+}\left|\Phi_{-}\right\rangle G_{\alpha_{1}}^{+} G_{\alpha_{2}}^{+} G_{\alpha_{3}}^{+}\left|\Phi_{-}\right\rangle .
\end{aligned}
$$

To conclude this section, the $n$-point function will be expressed in terms of $V$ and its spectral representation. From (1.8) it follows that

$$
\left\langle\sigma(\boldsymbol{r})_{n}\right\rangle_{t, N M}=Z_{t}^{-1} \operatorname{Tr}\left(V^{x_{1}} \sigma_{y_{1}}^{x} V^{x_{2}-x_{1}} \sigma_{y_{2}}^{x} \ldots V^{x_{n}-x_{n-1}} \sigma_{y_{n}}^{x} V^{N-x_{n}}\right)
$$

with

$$
Z_{t}=\operatorname{Tr} V^{N}
$$

The thermodynamic limit of the $n$-point function is known to exist in the sense of van Hove [16]. In (2.38) one can then take $N \rightarrow \infty$ followed by $M \rightarrow \infty$. Since the maximum eigenvalue of $V, \Lambda_{+}$, is strictly non-degenerate for finite $M$ by the Perron-Frobenius theorem [17], vector $\left|\Phi_{+}\right\rangle$, it follows that

$$
\left\langle\sigma(r)_{n}\right\rangle=\lim _{M \rightarrow \infty}\left\langle\Phi_{+}\left|\sigma_{y_{1}}^{x} \hat{V}^{x_{2}-x_{1}} \ldots \hat{V}^{x_{n}-x_{n-1}} \sigma_{y_{n}}^{x}\right| \Phi_{+}\right\rangle,
$$

where $\hat{V}=V / \Lambda_{+} ; \hat{V}$ has maximal eigenvalue unity. 
Spectral decomposition of $\hat{V}$ gives

$$
\begin{aligned}
& \left\langle\sigma(\boldsymbol{r})_{n}\right\rangle=\lim _{M \rightarrow \infty} \sum_{j_{1} \ldots j_{n-1}} \exp -\sum_{1}^{n}\left[\gamma_{j}\left(x_{j+1}-x_{j}\right)-i \omega_{j}\left(y_{j+1}-y_{j}\right)\right] \\
& \left\langle\Phi_{+}\left|\sigma_{1}^{x}\right| \Phi_{j_{1}}\right\rangle \prod_{1}^{n-1}\left\langle\Phi_{j_{l}}\left|\sigma_{1}^{x}\right| \Phi_{j_{l+1}}\right\rangle\left\langle\Phi_{j_{n-1}}\left|\sigma_{1}^{x}\right| \Phi_{+}\right\rangle .
\end{aligned}
$$

In the above $V$ and $T$ have been brought to diagonal form simultaneously and

$$
T\left|\Phi_{j}\right\rangle=e^{i \omega_{j}}\left|\Phi_{j}\right\rangle, \quad \hat{V}\left|\Phi_{j}\right\rangle=e^{-\gamma_{j}}\left|\Phi_{j}\right\rangle
$$

describes the translational character of the states. Thus, as stated previously, the objective is to determine all the matrix elements which occur in (2.41). By using the linear dependence in (2.29) and the defining relationships (2.9), (2.14), and (2.28) this will have been achieved when $\left\langle\Phi_{-}\left|G_{\alpha_{n}} \ldots G_{\alpha_{1}}\right| \Phi_{+}\right\rangle$has been obtained.

The key to the next section is the observation that

$$
\left\langle\Phi_{-}\left|G_{\alpha_{n}} \ldots G_{\alpha_{2}} G_{\beta}\right| \Phi_{+}\right\rangle=0 .
$$

Using the linear dependence of the $G_{\beta}$ on $G_{\alpha}$ and $G_{\alpha}^{+},(2.43)$ gives an integral recurrence relation on $n$ for the matrix elements which can be solved by an ansatz of the Pfaffian type [18] which is therefore reminiscent of Wick's theorem [19]. The analytic development for operators $G$ defined by $(2.28)$ will now be given. As the reader will perceive, the underlying translational invariance enters in an essential way in the following analysis of (2.43).

\section{General Method}

This section begins with some definitions, labelled for convenience $D j$, which will be referred to in what follows.

D1: $(z)_{n}$ is the $n$-tuple of complex numbers $z_{j}$;

$$
(z)_{n}=\left(z_{1}, \ldots, z_{n}\right) \text {. }
$$

The notation $(z)_{n m}, 1 \leqq n<m$ denotes

$$
(z)_{n m}=\left(z_{n}, \ldots, z_{m}\right) \text {. }
$$

The special case $(z)_{0}$ will be regarded as the empty set $\emptyset$. Obviously $(z)_{I}$ can be defined for any subset $I$ of the positive integers.

D2: For $I \neq \emptyset$, we define $\Delta_{i}, i \in \mathbb{Z}^{+}$by

$$
\begin{aligned}
\Delta_{i}(z)_{I} & =(z)_{I \backslash i}, & & i \in I \\
& =\emptyset, & & i \notin I .
\end{aligned}
$$

Finally, for any $J \subset \mathbb{Z}^{+}$, we have

$$
\Delta_{J}=\prod_{i \in J} \Delta_{i}
$$


D3: Complex-valued functions $F\left((z)_{n}\right)$ are defined for $n \geqq 1$. To exploit the $\Delta$ notation above fully, $F\left((z)_{0}\right)=F(\emptyset)$ is taken to be a complex constant.

D4.: Let

$$
F_{M}\left((z)_{n}\right)=M^{n / 2} \exp i \sum_{1}^{n}\left(\alpha_{j}+\theta\left(\alpha_{j}\right)\right)\left\langle\Phi_{-}\left|G_{\alpha_{n}} \ldots G_{\alpha_{1}}\right| \Phi_{+}\right\rangle
$$

with $z_{j}=\exp i \alpha_{j}$.

D5: Two classes of functions $\Theta(z)$, introduced in (2.32), will be considered:

$\mathscr{A}: \Theta\left(e^{i \omega}\right) \in \mathbb{L}^{2}([0,2 \pi]), \quad \Theta(z) \quad$ is

continuous and non-vanishing on $|z|=1$.

$\mathscr{A}_{r}: \Theta(z)$ is analytic in an anular region

$\left\{z: r^{-1}<|z|<r\right\}$ with $r>1$.

The winding number

$\mathscr{I}(\Theta)$ is given by

$$
\mathscr{I}(\Theta)=\Delta_{C}(\arg \Theta) / 2 \pi
$$

where $\Delta_{C}$ is the increment entailed by one positive passage around the unit circle.

Proposition 3.1. For $n \geqq 2$, the $F_{M}\left((z)_{n}\right)$ of D4, Equation (3.4) satisfy

$$
\begin{gathered}
\frac{2}{M} \sum_{z_{1} \in S_{M}^{+}} F_{M}\left((z)_{n}\right) \frac{1}{z_{1} / t-1}\left(1+\frac{\Theta\left(z_{1}\right)}{\Theta(t)}\right) \\
=\sum_{2}^{n}(-1)^{j} h\left(t, z_{j}\right) F_{M}\left(\Delta_{1 j}(z)_{n}\right),
\end{gathered}
$$

where

$$
h(t, z)=\frac{2 z t}{z t-1}\left(\frac{1}{\Theta(z) \Theta(t)}-1\right) .
$$

When $n=1$, the right hand side of (3.7) is replaced by zero. The function $F_{M}\left(z_{1}\right)$ must satisfy the additional equation

$$
\frac{1}{M} \sum_{z \in S_{M}^{+}} \Theta(z) F(z)=\left\langle\Phi_{-}\left|\sigma_{1}^{x}\right| \Phi_{+}\right\rangle .
$$

Proof. Use the vacuum property $\left\langle\Phi_{-}\left|G_{\alpha_{n}} \ldots G_{\alpha_{1}}\right| \Phi_{+}\right\rangle$, the linear dependence relationship (3.32) and the anti-commutation relation $\left[G_{\alpha_{1}}^{+}, G_{\alpha_{2}}\right]_{+}=\delta_{\alpha_{1} \alpha_{2}}$. Introducing (2.23), (2.24) and (2.33) together with (3.4) gives (3.6) and (3.7). The result (3.8) follows from (2.31), (2.17), and (2.12).

Proposition 3.2. If $\Theta \in \mathscr{A}(r)$ we have

$$
\begin{gathered}
\frac{1}{\pi i} \oint_{C} \frac{d z_{1}}{z_{1}} \frac{z_{1}^{M}}{z_{1}^{M}-1}\left(1+\frac{\Theta\left(z_{1}\right)}{\Theta(t)}\right) F_{M}\left((z)_{n}\right) \\
=\sum_{2}^{n}(-1)^{j} h\left(t, z_{j}\right) F_{M}\left(\Delta_{1 j}(z)_{n}\right),
\end{gathered}
$$


where $C$ is a simple closed contour in the anulus of analyticity surrounding the zeros of $z_{1}^{M}-1$, but not the pole at $z_{1}=t$ (Note: $t^{M}=-1$ ).

Proof. Use the residue theorem and the fact that $F_{M}\left((z)_{n}\right)$ is a product of a function analytic in $z_{1}$ for $r^{-1}<\left|z_{1}\right|<r$ and a polynomial of degree $2 M$.

Remarks. Proposition 3.1 suggests that one solution of (3.6) might be given in terms of the functions $f_{M}(z, t)$ which satisfy (3.6) with $n=2$. This would give a recurrence relation with $\left\langle\Phi_{-} \mid \Phi_{+}\right\rangle$and $F_{M}(z)$ as the initial conditions for $n$ even and odd respectively. But Proposition 3.2 suggests that, since interest only attaches to the limit $M \rightarrow \infty,(3.9)$ should be replaced by

$$
\left(Y_{+} F\right)\left((z)_{n}\right)=\sum_{2}^{n}(-1)^{j} h\left(z_{1} z_{j}\right) F\left(\Delta_{1 j}(z)_{n}\right)
$$

with the operator $Y_{+}$defined in principle by

$$
\left(Y_{+} F\right)\left((z)_{n}\right)=\frac{\mathscr{P}}{\pi i} \int_{C_{1}} \frac{d t}{t} \frac{1}{t / z_{1}-1}\left(1+\frac{\Theta(t)}{\Theta\left(z_{1}\right)}\right) F\left(t,(z)_{2, n}\right),
$$

where $C_{1}$ is the positive unit circle and $\mathscr{P}$ is the Cauchy principal part. Thus one poses the following problem: Let $F\left((z)_{n}\right) \in \mathscr{A} \bigotimes_{1}^{n}\left(L^{2}\left(S_{1}\right)\right)$ be a function on the $n$-particle subspace of Fock space [20]. Then (3.11) is properly defined (by reference to the theory of the Hilbert transform [21]). What solutions exist to (3.10) and in what sense are they approximated by the solution of (3.9)? The following two sections are addressed to these points.

\section{A Wick Theorem}

The operator $Y_{+}$defined by (3.11) was first encountered by Yang [22]. Properly speaking, it is only defined by (3.11) on a dense subset of the Hilbert space $\bigotimes_{1}^{n} L^{2}\left(S_{1}\right)$. When $n=1$, the following two propositions may be verified.

Proposition 4.1. i) $\left\|Y_{+}\right\| \leqq 2$;

ii) $Y_{+}$is self-adjoint (provided $\Theta(\omega)$ is real);

iii) one has the decomposition

$$
L^{2}\left(S_{1}\right)=\mathscr{B}_{0} \oplus \mathscr{B}_{0}^{\perp},
$$

where $\mathscr{B}_{0}$ is the invariant subspace with zero eigenvalue.

Proposition 4.2. Let $f, g \in L^{2}\left(S_{1}\right)$; consider the solutions of $Y_{+} f=g$. If $\mathscr{B}_{0}=\{0\}$ then $Y_{+}$is invertible. On the other hand, if $\operatorname{dim} \mathscr{B}_{0}>0$ then a solution only exists if $g \in \mathscr{B}_{0}^{\perp}$ : then

$$
f=\hat{Y}_{+}^{-1} g+h,
$$

where $\hat{Y}_{+}$is the restriction of $Y_{+}$to $\mathscr{B}_{0}^{\perp}$, and $h \in \mathscr{B}_{0}$ is arbitrary. 
By appealing to the theory of semi-infinite Töplitz forms a simple criterion can be given the dimension of $\mathscr{B}_{0}$ :

Theorem 4.1. Let $\Theta \in \mathscr{A}$ : then

$$
\operatorname{dim} \mathscr{B}_{0}=|\mathscr{I}(\Theta)| \text {. }
$$

The proof of this is given in Appendix A.

The following theorems discuss the solution of (3.11) in the cases $\mathscr{I}(\Theta)=0$ and $\mathscr{I}(\Theta)= \pm 1$

Theorem 4.2. If $\mathscr{I}(\Theta)=0$, then (3.10) and (3.11) have the unique solution

$$
\begin{aligned}
& F\left((z)_{2 n+1}\right)=0, \\
& F\left((z)_{2 n}\right)=\sum_{2}^{2 n}(-1)^{j} f\left(z_{1} z_{j}\right) F\left(\Delta_{1 j}(z)_{2 n}\right),
\end{aligned}
$$

where

$$
f(z, t)=\left(Y_{+}^{-1} h\right)(z, t) .
$$

The proof follows immediately from Proposition 4.2.

Remarks. 1. In the Appendix B, Wiener-Hopf factorisation is used to show that

$$
f(z, t)=\frac{z t}{z t-1}\left(\Theta_{+}^{-1}(z) \Theta_{-}^{-1}(t)-\Theta_{+}^{-1}(t) \Theta_{-}^{-1}(z)\right),
$$

where

$$
\Theta=\Theta_{+} \Theta_{-}
$$

is a canonical Wiener-Hopf factorisation (see Appendix B, Definition B.1). Note that $f(z, t)$ is antisymmetric and, with $|t|=1$, is $z$-analytic for $r^{-1}<|z|<r$.

2. The solution (4.5) is just the defining relation for a Pfaffian with pair contraction $f$ [18]. Since $f$ is antisymmetric, $F\left((z)_{2 n}\right)$ is also antisymmetric in its arguments, as anticipated.

3. The recurrence (4.5) has the boundary condition $F(\emptyset)$ which has to be determined by other methods.

Theorem 4.3. If $\mathscr{I}(\Theta)= \pm 1$ and $\Theta \in \mathscr{A}$, then a solution of (3.11) is

$$
F\left((z)_{2 n+1}\right)=\sum_{2}^{2 n+1}(-1)^{j} f\left(z_{1} z_{j}\right) F\left(\Delta_{1 j}(z)_{2 n+1}\right)+F\left(z_{1}\right) G\left((z)_{2,2 n+1}\right)
$$

and

$$
F\left((z)_{2 n}\right)=\sum_{2}^{2 n}(-1)^{j} f\left(z_{1} z_{j}\right) F\left(\Delta_{1 j}(z)_{2 n}\right)
$$

where

$$
\left(Y_{+} F\right)(z)=0
$$


and

$$
\begin{aligned}
\frac{1}{2 \pi i} \oint_{C_{1}} \frac{d z}{z} \Theta(z) F(z) & =\lim _{M \rightarrow \infty}\left\langle\Phi_{-}\left|\sigma_{1}^{x}\right| \Phi_{+}\right\rangle \\
& =m^{*} .
\end{aligned}
$$

The function $G$ is arbitrary and $f(z, t)$ is a solution of the equation

$$
\left(Y_{+} f\right)(z, t)=h(z, t)+F(t) G(z) .
$$

Proof. Equations (4.9) and (4.13) constitute a solution of (3.11) because the term $F(t)$ in (4.13) gives a Pfaffian with two repeating rows; this vanishes. The initial condition for (4.9), $F(z)$, should be fixed by (4.12). Equation (4.10) is a solution analogous to that of Theorem (4.2) with $F(\emptyset)$ as an initial condition.

Remarks. 1. Using the Wiener-Hopf factorisations given in Appendix B, the following results are readily obtained [see (4.12)]: if

$$
\begin{aligned}
& \mathscr{I}(\Theta)=1, \quad F(z)=m^{*} \Theta(0) \Theta_{-}^{-1}(z), \\
& \mathscr{I}(\Theta)=-1, \quad F(z)=m^{*} \Theta_{+}(0) z \Theta_{+}^{-1}(z)
\end{aligned}
$$

with $f(z, t)$ defined by

$$
f(z, t)=\frac{z t}{z t-1}\left(\Theta_{+}^{-1}(z) t^{-1} \Theta_{-}^{-1}(t)-\Theta_{+}^{-1}(t) z^{-1} \Theta_{-}^{-1}(z)\right)
$$

one has (4.13) with $F(t)=1 / \Theta(t)$ and

$$
G(z)=2 \Theta_{+}(0) z / \Theta_{+}(z)\left(\operatorname{resp} .2 \Theta_{-}(0) / \Theta_{-}(z)\right)
$$

for $\mathscr{I}(\Theta)=-1$ (resp. +1$)$.

Theorem 4.4. If $\mathscr{I}(\Theta)= \pm 1$, the unique antisymmetric solution of (3.10) in the form (4.9) and (4.16) is

$$
F\left((z)_{2 n+1}\right)=\sum_{1}^{2 n+1}(-1)^{j} F\left(z_{j}\right) F\left(\Delta_{j}(z)_{2 n+1}\right) .
$$

Proof. The antisymmetry of (4.18) follows from basic manipulation. The proof of uniqueness proceeds by induction: $F\left(z_{1}\right)$ is certainly unique (see Appendix A). Make the inductive ansatz that $F\left((z)_{2 n-1}\right)$ is unique. Then, if $F_{1}\left((z)_{2 n+1}\right)$ and $F_{2}\left((z)_{2 n+1}\right)$ are two solutions of (3.10) in terms of $F(z)_{2 n-1}$, it follows that

$$
Y_{+}\left(F_{1}-F_{2}\right)=0
$$

which has the solution

$$
\left(F_{1}-F_{2}\right)(z)_{2 n+1}=F\left(z_{1}\right) G\left((z)_{2,2 n+1}\right) .
$$

But $F_{1}-F_{2}$ must also be antisymmetric. Hence $G=0$ a.e. and the theorem is proved. 


\section{Thermodynamic Limit}

We now return to the question of the sense in which $F\left((z)_{n}\right)$ is a limit of the $F_{M}\left((z)_{n}\right)$ which satisfy (3.9). A solution to this equation certainly exists in the Pfaffian form of (4.9) and (4.9) but with an $M$-dependent contraction function $f_{M}(z, t)$ and, if $\mathscr{I}(\Theta)= \pm 1$, and $M$-dependent element in $\mathscr{B}_{0}$. We now construct an integral equation for the difference

$$
\left(\delta f_{M}\right)(z)_{2}=f\left((z)_{2}\right)-f_{M}\left((z)_{2}\right) .
$$

If $\Theta \in \mathscr{A}(r)$ then (3.10) and (3.11) may be rewritten as

$$
\frac{1}{\pi i} \oint_{C_{+}} \frac{d z_{1}}{z_{1}} \frac{1}{z_{1} / t-1}\left(1+\frac{\Theta\left(z_{1}\right)}{\Theta(t)}\right) f\left((z)_{2}\right)-2 f\left(t, z_{2}\right)=h\left(t, z_{2}\right)
$$

where $C_{+}=\left\{z:|z|=r^{1}, 1 \leqq r^{1}<r\right\}$ taken once in the positive sense. Taking (5.2) with (3.9) gives

$$
\left(Y_{+} \delta f_{M}\right)(z)_{2}=\mathscr{E}_{M}\left((z)_{2}\right),
$$

where $\mathscr{E}_{M}$ is given by

$$
\begin{aligned}
\mathscr{E}_{M}\left((z)_{2}\right)= & \frac{1}{\pi i}\left\{\oint_{C_{+}} \frac{d t}{t} \frac{1}{t^{M}-1}-\oint_{C_{-}} \frac{d t}{t} \frac{t^{M}}{t^{M}-1}\right\} \\
& \cdot \frac{1}{t / z_{1}-1}\left(1+\frac{\Theta(t)}{\Theta\left(z_{1}\right)}\right) f_{M}\left(t, z_{2}\right),
\end{aligned}
$$

where $C_{-}=\left\{z:|z|=r^{\prime}, r^{-1}<r^{\prime}<1\right\}$, again taken once in the positive sense. Now $f_{M}\left(t, z_{2}\right)$ is analytic in $t$ for $r^{-1}<|t|<r$. Hence

$$
\left|\mathscr{E}_{M}\left((z)_{2}\right)\right| \leqq b r_{0}^{-M}\left(\underset{C_{+}}{\oint_{C_{-}}}+\oint_{C^{\prime}}\right)\left|f_{M}\left(t, z_{2}\right)\right|^{2} d t
$$

by use of the Cauchy-Schwartz inequality. Since $f_{M}\left(t, z_{2}\right)^{*}$ is also analytic in $t$ for $r^{-1}<|t|<r,\left|z_{2}\right|=1$, the line integrals in (5.4) may be deformed to the unit circle. Now $\left\|f_{M}\right\|_{2} \leqq 1$. Thus

$$
\left\|\delta f_{M}\right\|_{2} \leqq 2\left\|Y_{+}^{-1}\right\| b r_{0}^{-M} \text {, }
$$

where $1<r_{0}<r$. This bound will be satisfactory. If $\mathscr{B}_{0}$ is non-null then the restriction of $Y_{+}$to $\mathscr{B}_{0}^{\perp}$ should be taken. Precisely the same sort of bounding procedure works for $\delta F(z)$.

The following two lemma show how the estimates are applied in establishing the thermodynamic limit.

Lemma 5.1. The matrix elements satisfy the bound

$$
\left\|F_{M}\left((z)_{2 n}\right)-F\left((z)_{2 n}\right)\right\| \leqq(2 n(2 n-2) \ldots 2)\left\{\exp \left(n\left\|\delta f_{M}\right\| /\|f\|\right)^{-1}\right\} .
$$

Proof. Consider the Pfaffian expansion; replace each contraction $f_{M}(\cdot, \cdot)$ by $f(\cdot, \cdot)+\delta f_{M}$. Each term has an $L^{2}$ bound

$$
(\|f\|+\|\delta f\|)^{n} \leqq\|f\|^{n} \exp n \log (1+\|\delta f\| /\|f\|)
$$


from which (5.6) follows. The prefactor is just the number of terms in the Pfaffian expansion.

Lemma 5.2. Suppose that for a sequence $\left\{G\left((\omega)_{n}\right) ; n \geqq 0\right\}$ of $\bigotimes_{1}^{n} L^{2}[0,2 \pi]-$ integrable functions there exists a constant $K>0$ such that

$$
\sup _{n \geqq 0} \underset{(\omega)_{n}}{\operatorname{ess}} \sup \mid G\left((\omega)_{n}\right) e^{n K}=C<\infty .
$$

Then

$$
\begin{aligned}
\lim _{M \rightarrow \infty} & \sum_{0}^{\infty} \frac{1}{n !(2 \pi)^{n}} \int_{-\pi}^{\pi} \int_{-\pi} d(\omega)_{n}\left|F_{M}\left(\left(e^{i \omega}\right)_{n}\right)\right|^{2} G\left((\omega)_{n}\right) \\
& =\sum_{0}^{\infty} \frac{1}{n !(2 \pi)^{n}} \int_{-\pi}^{\pi} \int_{\pi} d(\omega)_{n}\left|F\left(\left(e^{i \omega}\right)_{n}\right)\right|^{2} G\left((\omega)_{n}\right) .
\end{aligned}
$$

Proof. This follows directly from Lemma 5.1, its obvious extension to the case $n$ odd, and from (5.7)

The following lemma is a trivial consequence.

Lemma 5.3. Analogous estimates to those of Lemmas 5.1 and 5.2 hold with discrete sums $\omega_{j}=2 \pi j / M, j=1, \ldots, M$.

These results suffice to establish the thermodynamic limit. The existence of limits term by term in $n$ is a direct consequence of the Helly theorems [25].

\section{Conclusions}

This paper has given a general method for handling odd operators in spinor algebras of the type encountered in lattice statistics. It relies on the linear dependence between the two families to produce the perhaps unexpected Wick structure. The cyclic symmetry enters in an essential way; the reader will notice that this leads to semi-infinite Töplitz forms whose properties are rather well known and very useful in this context. The following papers shows how the ideas developed here can be applied to the rectangular Ising model.

Acknowledgements. The author thanks the Aspen Center for Physics, the I.H.E.S., the Lab. de Physique Theorique of the EPF-Lausanne and the Rockefeller University for hospitality and support while parts of this work were carried out. He is most grateful to Professors P. L. Choquard, M. L. Glasser, J. Glimm, J. Groeneveld, M. Kac, H. Kunz, and T. Spencer for discussions.

\section{Appendix A: Semi-Infinite Töplitz Forms}

First consider the content of the subspace $\mathscr{B}_{0}: Y_{+} f=0$ can be rewritten as

$$
\frac{\mathscr{P}}{\pi i} \int_{C_{1}} \frac{d z}{z-t}\left(1+\frac{\Theta(z)}{\Theta(t)}\right) g(z)=0,
$$

where $g(z)=f(z) / z$. 
Introducing the Hilbert transform on $L^{2}\left(S_{1}\right)$ by

$$
(H f)(t)=\frac{\mathscr{P}}{\pi i} \int_{\mathrm{C}_{1}} \frac{d z}{z-t} f(z)
$$

reduces (A.1) to the form

$$
\Theta H g+H \Theta g=0 \text {. }
$$

Now $\Theta$ and $g$ have $L^{2}$-convergent Fourier series. The coefficients for a function $f$ are defined by

$$
f\left(e^{i \omega}\right)=\sum_{-\infty}^{\infty} f_{n} e^{i n \omega} .
$$

From (A.2), we can equivalently regard $H$ as operating on the associated $l^{2}$ space of the coefficients:

$$
(H f)_{n}=f_{n} \operatorname{sgn} n .
$$

Then (A.3) takes the form

$$
\begin{aligned}
& \sum_{0}^{\infty} \Theta_{n-m} f_{m}=0 \\
& \sum_{0}^{\infty} \Theta_{n-m}^{-1} f_{(-(m+1))}=0
\end{aligned}
$$

for $0 \leqq n<\infty$. From the Definition D.5, (3.5) the winding number satisfies

$$
\mathscr{I}\left(\Theta^{-1}\right)=-\mathscr{I}(\Theta) \text {. }
$$

Definition. D. A.1.: A semi-infinite Töplitz form is defined for $\Theta \in L^{2}\left(S_{1}\right)$ for $n$ a non-negative integer in terms of

$$
\left(T_{\Theta} f\right)_{n}=\sum_{0}^{\infty} \Theta_{n-m} f_{m} .
$$

The following theorem is essential to our thinking:

Theorem A1. Let $\Theta\left(e^{i \omega}\right)$, be continuous in $\omega$. Then $T_{\Theta}$ is invertible if and only if $\Theta(z) \neq 0$ for $|z|=1$ and $\mathscr{I}(\Theta)=0$. For the proof, see [23].

Remark. The following remarks are addressed to the inversion of $T_{\Theta}$ when $\mathscr{I}(\Theta)=0$, and to the construction of $\mathscr{B}_{0}$ when $\mathscr{I}(\Theta) \neq 0$.

Definitions. D.A.2.: Let $f \in L^{2}\left(S_{1}\right)$ : then $f$ is analytic (resp. coanalytic) if $C_{n}=0$ for $n<0$ (resp. $n \geqq 0$ ).

Lemma A1. If $\Theta$ has the form

$$
\Theta=\Psi \Phi
$$

with either $\Phi$ of analytic type $0 \Psi$ of coanalytic type, then

$$
T_{\Theta}=T_{\Psi} T_{\Phi} .
$$


Lemma A2. Assume $\Phi$ is of analytic (resp. coanalytic) type. Then $T_{\Phi}$ is invertible if and only if $\Phi$ is bounded away from zero inside (resp. outside) the unit circle.

Lemma A3. If $\Phi=\Phi_{+} \Phi_{-}$where $\Phi_{+}$(resp. $\Phi_{-}$) is analytic (resp. coanalytic) and $\Phi_{ \pm}$satisfy the boundedness conditions in Lemma A2. Then

$$
T_{\Phi}^{-1}=T_{1 / \Phi_{+}} T_{1 / \Phi_{-}} .
$$

The cases with $\mathscr{I}(\Theta)= \pm n, n \in \mathbb{Z}^{+}$can now be handled.

1. $\mathscr{I}(\Theta)=n, n>0$. Define

$$
\hat{\Theta}(z)=z^{n} \Theta(z)
$$

so that $\mathscr{I}(\hat{\Theta})=0$ using Lemma A1, it follows that

$$
T_{\Theta}=\left(S^{*}\right)^{n} T_{\hat{\Theta}}
$$

and

$$
T_{\Theta-1}=T_{\widehat{\Theta}-1} S^{n}
$$

where the shift $S$ is defined by

$$
(S f)_{0}=0, \quad(S f)_{n}=f_{n-1}, \quad n \geqq 1
$$

and $S^{*}$ is its adjoint

$$
\left(S^{*} f\right)_{n}=f_{n+1} \text {. }
$$

Now let $B$ by any $l_{+}^{2}$ vector such that $\left(T_{\hat{\Theta}} b\right)_{m}=0$ for all $m \geqq n-1$.

Then it follows from that

$$
T_{\Theta} b=0 .
$$

However, the equation

$$
T_{\Theta-1} a=0
$$

has only the solution $a=0$.

2. $\mathscr{I}(\Theta)=-n, n \in \mathbb{Z}^{+}$. Define

$$
\hat{\Theta}(z)=z^{-n} \Theta(z) \text {. }
$$

Then we have

$$
T_{\Theta}=T_{\hat{\Theta}} S^{n}
$$

and

$$
T_{\Theta^{-1}}=\left(S^{*}\right)^{n} T_{\hat{\Theta}^{-1}} .
$$

In this case, $T_{\Theta} a=0$ has only the solution $a=0$ whereas

$$
T_{\Theta-1} b=0
$$

has $n$ linearly independent solutions. 


\section{Appendix B: Wiener-Hopf Factorisation}

Solutions of Equations (4.6) and (4.13) can be given in closed form in terms of the Wiener-Hopf factorisation of the function $\Theta(z)$. In this appendix only the cases $\mathscr{I}(\Theta)=0, \pm 1$ will be considered. The necessary theorems and definitions can be found in the article by Krein [24]:

Definition B.1. If $\Theta\left(e^{i \omega}\right) \in L^{2}([0,2 \pi])$ the representation

$$
\Theta(z)=\Theta_{+}(z) \Theta_{-}(z)
$$

is a canonical factorisation if $\Theta_{+}$(resp. $\Theta_{-}$) is analytic and non-vanishing for $|z|<1$ (resp. $|z|>1)$.

Theorem B1. (Theorem 5.1 of [24]): For a function $\Theta\left(e^{i \omega}\right) \in L^{2}([0,2 \pi])$ to admit of a canonical factorisation, it is necessary and sufficient that $\Theta(z) \neq 0$ if $|z|=1$ and that $\mathscr{I}(\Theta)=0$, i.e. $\Theta \in \mathscr{A}$ of Definition D6.

Remarks. 1. The factorisations can be computed from the formulae

$$
\begin{array}{ll}
\log \Theta_{+}(z)=\frac{1}{2 \pi i} \oint_{C_{1}} \frac{d \mathscr{G}}{\mathscr{G}-z} \log \Theta(\mathscr{G}), & |z|<1 \\
\log \Theta_{-}(z)=-\frac{1}{2 \pi i} \oint_{C_{1}} \frac{d \mathscr{G}}{\mathscr{G}-z} \log \Theta(\mathscr{G}), \quad|z|>1 .
\end{array}
$$

2. Since $\Theta\left(e^{i \alpha}\right)=\Theta^{-1}\left(e^{-i \alpha}\right)$ for $\alpha \in \mathbb{R}$, (B.2) requires that

$$
\Theta_{+}\left(z^{-1}\right)=\Theta_{-}^{-1}(z) \text {. }
$$

3. From Appendix $\mathrm{A}$ it is known that (4.6) has a unique solution if $\mathscr{I}(\Theta)=0$. This solution is then readily seen to be

$$
f(z, t)=\frac{z t}{z t-1}\left(\Theta_{+}^{-1}(z) \Theta_{-}^{-1}(t)-\Theta_{+}^{-1}(t) \Theta_{-}^{-1}(z)\right) .
$$

Theorem B2. If $\mathscr{I}(\Theta)=+1$ (resp. -1) then there exists a factorisation of type (B.1) which is canonical, except that $\Theta_{+}(z)$ (resp. $\left.\Theta_{-}(z)\right)$ has a simple zero at $z=0$ (resp. $z=\infty)$.

Remarks. 1. By considering $z^{ \pm 1} \Theta(z)$ and (B.3) it follows that

$$
z \Theta_{+}\left(z^{-1}\right)=1 / \Theta_{-}(z) \text {. }
$$

2. It can easily be checked that the function $f(z, t)$ given by (4.16) does satisfy

\section{References}

1. Onsager,L.: Phys. Rev. 65, 117 (1944)

2. Onsager,L., Kaufman, B.: Phys. Rev. 76, 1244 (1949)

3. Schultz,T.D., Mattis,D.C., Lieb, E.H.: Rev. Mod. Phys. 36, 856 (1964)

4. McCoy, B.M., Wu,T.T.: The two-dimensional Ising model. Cambridge, Massachusetts: Harvard University Press 1973 
5. Abraham,D.B.: Phys. Lett. 61 A, 271 (1977)

6. McCoy,B.M., Tracy,C.A., Wu,T.T.: Phys. Rev. Letters 38, 793 (1977)

7. An Yang, H.: Preprint, SUNY, Stony Brook

8. Abraham,D.B. : Phys. Letters 39 A, 357 (1972). Details to be published in the fourth of this series of papers

9. Abraham,D. B., Reed,P.: Phys. Rev. Letters 33, 377 (1974); Commun. math. Phys. 39, 35 (1976); J. Phys. 10 A, L121 (1977)

10. Gallavotti,G. : Nuovo Cimento 2, 133 (1972)

11. See References in [3]

12. Lewis,J.T., Sisson,P.N.M.: Commun. math. Phys. 44, 279 (1975)

13. Abraham,D.B., McLachlan, A.D.: Molec. Phys. 12, 301 (1967). This is a special case of imposition of boundary conditions in the Bethe ansatz; see Yang, C. N., Yang, C.P.: Phys. Rev. 150, 321 (1966)

14. Bogoliubov, N.N.: Usp. Fiz. Nauk. 67, 549 (1959) Valatin,J.G.: Phys. Rev. 122, 1012 (1961)

15. Kac, M.: Brandeis lectures in theoretical physics. New York: Gordon and Breach 1966

16. Ruelle,D.: Statistical mechanics. New York: Benjamin 1969

17. Bellman, R.: Introduction to matrix analysis. New York: McGraw Hill 1960. See also Heilmann,O.J.: Matrix theory (to be published)

18. Caianiello,E.R.: Combinatorics and renormalisation in quantum field theory. New York: Benjamin 1973

19. Wick, G.C.: Phys. Rev. 80, 268 (1950)

20. Dubin,D. A. : Solvable models in algebraic statistical mechanics. Oxford: Oxford University Press 1974

21. Dym,H., McKean,H.P.: Fourier series and integrals. New York: Academic Press 1972

22. Yang, C. N. : Phys. Rev. 85, 808 (1952)

23. Widom,H.: Stud. Math. 3, 179 (1965)

24. Krein,M.G.: Usp. Mat. Nauk (N.S.) 13, No. 5 (83), 3-120 (1958). English translation: A.M.S. Translations, Series 2, 22, 163 (1962)

25. Burrill,C.W. : Measure, integration and probability. New York: McGraw-Hill 1972

Communicated by E. Lieb

Received November 29, 1977

\section{Note Added in Proof}

Considerable recent progress has been made independently of [6] and the work of this author by Bariev, R. Z.: Phys. Lett. 64A, 169 (1977). 